\title{
Phenomena, theory, application, data, and methods all have impact
}

\author{
John R. Hauser ${ }^{1}$
}

Published online: 19 September 2016

(C) Academy of Marketing Science 2016

\section{The importance of phenomena}

In his provocative essay on impactful research, my colleague and friend Gerry Tellis (2016) postulates that good papers are interesting and challenge common beliefs. He postulates further that such papers are based on ideas that are simple once proposed, although not always so obvious before being proposed. He recommends that impactful papers be focused and brief and begin with a study of the basic phenomena. Great advice.

A study of phenomena has often informed my own research and, as an editor, I valued phenomenological research. However, my experience, as an editor, an advisor, an author, and a student of the history of science, suggests that there are many paths to impactful research. Some researchers focus on one or a few paths; others are more eclectic in their pursuits. The best researchers challenge established beliefs in a variety of ways. Before I discuss other paths, I'll add to Gerry's examples:

- In the late 1970s, simulated test markets were revolutionizing new product development. No firm would dare launch a new consumer packaged good without first testing it with a simulated test market. But the largest use of simulated test markets was by competitive firms who wanted to defend their turf. The next research topic was obvious - defensive marketing strategy.

John R. Hauser

hauser@mit.edu

1 MIT Sloan School of Management, Massachusetts Institute of Technology, E62-538, 77 Massachusetts Avenue,

Cambridge, MA 02142, USA
- As logit models began to be applied widely in marketing, it was not practical to model choice among all brands, particularly when there were 20 or more brands available. Researchers began to ask: "If it so hard to model consumer decisions, how do consumers actually make those decisions?" A closer examination by a variety of methods, from think-aloud protocols to information-theoretic analyses, suggested that consumers form consideration sets and choose from those consideration sets. This stylized fact led to new literatures in both behavioral and quantitative marketing.

- The study of consideration sets led researchers to ask how such sets were formed, which, in turn, led to a study of heuristic decision making and the constructed-preference hypothesis, and so on.

Initially, papers documented the phenomena; later, papers explored the limits of the stylized facts or explored generality across domains. From many papers and authors, generally accepted explanations evolved to provide broad-ranging theories - paradigms as defined by Kuhn (1970). Tenets of the theories are still being challenged today, leading to experiments that distinguish among competing explanations.

\section{The role of theory}

Gerry cites Thomas Kuhn's (1970) essay on the "Structure of Scientific Revolution." Kuhn argues persuasively that science advances through revolutions and that such revolutions often begin with phenomena. But Kuhn also talks about the role of theory in guiding what to study. By theory I do not mean a single hypothesis, but rather a sweeping worldview. Such theories provide systematic directions to identify critical experiments that produce rapid progress (Platt 1964). 
I'll use Gerry's example of Tycho Brahe's data, which were collected over 20 or more years-long before fiveto nine-year tenure clocks. His student, Johannas Kepler, synthesized the data to describe planetary motion with three simple laws. But neither Brahe nor Kepler were working without theory. They had both the sun-centered theory of Copernicus and the earth-centered theory of Ptolemy. The later had been the dominant theory for fifteen centuries. (Aristarchus' sun-centered system in the third century B.C. never caught on, perhaps because reviewers rejected his paper or perhaps because measurements of the time could not distinguish between the theories.) When Kepler did his research, neither theory was parsimonious; both theories required complex "kludges" to fit the data. ${ }^{1}$

Today we accept scientific consensus that the earth orbits the sun, not vice versa, but it was not always so obvious. In the seventeenth century, most scientists agreed that the sun-centered system had a fatal flaw-it could not simultaneously explain the lack of stellar parallax and the observed size of the stars (Haqq-Misra 2016). ${ }^{2}$ Kepler's theory was simpler and more elegant and could explain key phenomena, but Kepler's theory had the fatal flaw and could not replace the old theory immediately. The same is true today. Theories often guide research toward important experiments and observations. Consensus theories are ripe for challenge when they become too complex or when they cannot explain newly identified phenomena (Kuhn 1970, pp. 64-67).

To challenge existing beliefs, we need existing beliefs and we need research that pushes existing beliefs to their limits. Gerry's research on first-mover advantage was significant because it challenged scientific consensus. I am particularly enamored with a paper by Frederick et al. (2014). They noticed that the decoy effect was almost always tested on text-based examples rather than real products. They asked themselves: "What if we add a rotten apple to a choice set of an apple and an orange?" The decoy effect predicts the rotten apple should push consumers to the non-rotten apple, but common sense says the rotten apple will push consumers toward the orange. The authors provide evidence that this thought experiment is valid and generalizes. Their paper provides a fresh perspective on the decoy effect, but, more importantly, causes us to reexamine a wide range of behavioral phenomena.

Today there is a consensus that structural models of forward-looking consumers provide greater generalizability than "reduced-form" models. But, do consumers

\footnotetext{
${ }^{1}$ This is but a brief and simplified summary of the sequence documented by Kuhn (1970) and others.

${ }^{2}$ When I was an editor, if a reviewer pointed out a fatal flaw, I took it as a sign that the authors might be challenging existing dogma. The paper deserved another look.
}

really solve in their heads complex dynamic programs that are extremely hard to solve for even the fastest computers? To test that hypothesis we needed a product category that favored forward-looking behavior, and we needed to estimate both established models and reasonable heuristics (Lin et al. 2015). The evidence favors heuristics. That paper would be of little interest if it were not for the consensus favoring complex models of forward-looking behavior.

\section{Prescriptive research}

Marketing is an applied discipline; solving a relevant problem can have impact. ${ }^{3}$ For example:

- There are hundreds of published articles on conjoint analysis, a topic that began because managers wanted to select the features of new products based on consumer preferences. (By the way, the seminal paper by Paul Green and Vithala Rao was published only because the editor overruled the reviewers.)

- The first wide-spread application of Bayesian methods was in conjoint analysis. Lenk et al. (1996) solved the hitherto-unsolved practical problem of how to obtain heterogeneous partworths from shorter questionnaires. Later, adaptive questions selected by machine-learning methods improved applications further.

- Simulated test markets were developed because consumer-packaged-good test markets were costly and too slow. But simulated test markets were not feasible for really new products. The solution, information acceleration, was driven by auto companies who wanted to forecast the sales of electric vehicles, and wanted to do so before infrastructure, legislation, and climate concerns were prevalent.

Applications have led to research on auctions (driven by keyword advertising), multi-armed-bandit optimal experimentation (driven by the popularity of $\mathrm{A} / \mathrm{B}$ testing), website morphing (driven by the need to adapt websites to cognitive styles), and the study of social media.

\section{The role of data and new methods}

New data sources lead to impact. Gerry's example of firstmover advantage was made possible, in part, by the unique data that he and his collaborators collected. In

\footnotetext{
${ }^{3}$ Galileo's interest in the moons of Jupiter was driven, in part, by the need for mariners to be able to know their longitude at sea - one of the greatest practical and scientific challenges of the time (Sobel 1995, p. 14).
} 
the 1980 s, scanner data helped us understand consumer response to marketing tactics. In the early 2000 s, fast graphic computers and new algorithms revolutionized marketing research. Today, the availability of "big data" enables us to look at new phenomena, solve new problems, and propose new theory (Chintagunta et al. 2016). Many forms of user-generated content (Twitter, Facebook, blogs, reviews) are opening up new ways to understand and model consumers. And quality Internet panels are changing the way we do experiments.

New methods and capabilities also lead to impact. Logit models, Mouselab, Bayesian methods, machine learning, deep learning, and many other tools have opened or are opening new applications, new ways to identify phenomena, and new ways to test theory. There are, indeed, many paths to impactful papers. But whatever path you take, heed Gerry's advice to keep it interesting, simple, and brief.

\section{References}

Chintagunta, P., Hanssens, D., \& Hauser, J. R. (2016). Marketing science and big data. Marketing Science, 35(1), 1-2.

Frederick, S., Lee, L., \& Baskin, E. (2014). The limits of attraction. Journal of Marketing Research, 51, 487-507.

Haqq-Misra, J. (2016). The inquisition followed sound science. Boston Globe, August 7, K1 \& K4.

Kuhn, T. S. (1970). The structure of scientific revolutions, 2E. Chicago: University of Chicago Press.

Lenk, P. J., DeSarbo, W. S., Green, P. E., \& Young, M. R. (1996). Hierarchical Bayes conjoint analysis: recovery of partworth heterogeneity from reduced experimental designs. Marketing Science, 15, 173-191.

Lin, S., Zhang, J., \& Hauser, J. R. (2015). Learning from experience, simply. Marketing Science, 34(1), 1-19.

Platt, J. R. (1964). Strong inference. Science, 146, 347-53.

Sobel, D. (1995). Longitude: the true story of a lone genius who solved the greatest scientific problem of his time. New York: Walker and Company.

Tellis, G. (2016). Interesting and impactful research: on phenomena, theory, and writing. Journal of the Academy of Marketing Science. doi:10.1007/s11747-016-0499-0 